\title{
Effects of surface and interface traps on exciton and multi-exciton dynamics in core/shell quantum dots
}

Renato Bozio, Marcello Righetto, Alessandro Minotto

Renato Bozio, Marcello Righetto, Alessandro Minotto, "Effects of surface and interface traps on exciton and multi-exciton dynamics in core/shell quantum dots," Proc. SPIE 10348, Physical Chemistry of Semiconductor Materials and Interfaces XVI, 1034816 (24 August 2017); doi: $10.1117 / 12.2273713$

Event: SPIE Nanoscience + Engineering, 2017, San Diego, California, United States 


\title{
Effects of surface and interface traps on exciton and multi-exciton dynamics in core/shell quantum dots
}

\author{
Renato Bozio*a ${ }^{*}$ Marcello Righetto ${ }^{\mathrm{a}}$, Alessandro Minotto ${ }^{\mathrm{a}, \mathrm{b}}$ \\ ${ }^{a}$ Department of Chemical Sciences and R.U.-INSTM, University of Padova, Via Marzolo 1, I-35131 \\ Padova, Italy; ${ }^{\mathrm{b}}$ Department of Physics and Astronomy and London Centre for Nanotechnology, \\ University College London, Gower Street, London WC1E 6BT, United Kingdom
}

\begin{abstract}
Exciton interactions and dynamics are the most important factors determining the exceptional photophysical properties of semiconductor quantum dots (QDs). In particular, best performances have been obtained for ingeniously engineered core/shell QDs. We have studied two factors entering in the exciton decay dynamics with adverse effects for the luminescence efficiency: exciton trapping at surface and interface traps, and non-radiative Auger recombination in QDs carrying either net charges or multiple excitons. In this work, we present a detailed study into the optical absorption, fluorescence dynamics and quantum yield, as well as ultrafast transient absorption properties of $\mathrm{CdSe} / \mathrm{CdS}$, $\mathrm{CdSe} / \mathrm{Cd}_{0.5} \mathrm{Zn}_{0.5} \mathrm{~S}$, and $\mathrm{CdSe} / \mathrm{ZnS}$ QDs as a function of shell thickness. It turns out that de-trapping processes play a pivotal role in determining steady state emission properties. By studying the excitation dependent photoluminescence quantum yields (PLQY) in different $\mathrm{CdSe} / \mathrm{Cd}_{\mathrm{x}} \mathrm{Zn}_{1-\mathrm{x}} \mathrm{S}(\mathrm{x}=0,0.5,1) \mathrm{QDs}$, we demonstrate the different role played by hot and cold carrier trapping rates in determining fluorescence quantum yields. Finally, the use of global analysis allows us untangling the complex ultrafast transient absorption signals. Smoothing of interface potential, together with effective surface passivation, appear to be crucial factors in slowing down both Auger-based and exciton trapping recombination processes.
\end{abstract}

Keywords: Quantum Dots, Excitons, Photophysics, Trapping, Auger recombination

\section{INTRODUCTION}

Semiconductor nanocrystals - hereafter indicated as quantum dots (QDs) - present profitable opportunities for applications in many and diverse fields of biomedicine, energy and information technologies. ${ }^{1-6}$ Besides, QDs are rapidly spreading into the display and lighting markets. Compared with liquid crystal and organic LED displays, QDs provide highly saturated colors, wide color gamut, resolution, rapid response time, optical efficiency, durability and low cost. Both in lighting and photovoltaic technologies implementation of QDs occurs, most often, in the aggregate forms of QD layered solids or 3D supercrystals.

This remarkable progress has been made possible by the rapid advances in the synthesis of colloidal QDs and by the deepened understanding of the intriguing new physics exhibited by these nanoparticles. Most recent contributions to understanding the intrinsic photophysical properties of QDs come from single nanoparticle studies ${ }^{7}$ and ultrafast time resolved experiments. ${ }^{8,9}$ The issues addressed by these studies focus mainly on the role of exciton trapping at surface and interface traps, non radiative Auger recombination in QDs carrying either net charges or multiple excitons, and the emission intermittencies, a.k.a. blinking. Other phenomena, like energy and charge transfer, are key for lighting and energy applications and can be studied only as ensemble properties of QD aggregates.

Suitably engineered core/graded-shell QDs exhibit exceptionally favorable optical properties, photoluminescence and optical gain, while keeping the synthesis facile and producing QDs well suited for light emitting applications. Solid-state laser emitters can greatly profit from QDs as efficient gain materials. In the field of display technologies, the exploitation of the exceptional photoluminescence properties of QDs for LCD backlighting has already advanced to commercial levels. The next big challenge is to develop the electroluminescence properties of QD to a similar state. In fact, advances in this display technology are expected from the development of QD-based light emitting diodes (QLED) currently underway. ${ }^{10-13}$

Physical Chemistry of Semiconductor Materials and Interfaces XVI, edited by Hugo A. Bronstein,

Felix Deschler, Artem A. Bakulin, Proc of SPIE Vol. 10348, 1034816 · C) 2017

SPIE $\cdot$ CCC code: $0277-786 \mathrm{X} / 17 / \$ 18 \cdot$ doi: 10.1117/12.2273713 
However, further efforts are required to progress in our understanding of energy and charge transfer processes in QD films to ensure higher external efficiencies and long term stabilities. The choice of QDs to be used in QLEDs must consider high photoluminescence in dispersed solutions as a starting criterion, which means low trap densities and reduction of Auger recombination. However, in QD films the role of recombination at surface traps is amplified because energy transfer brings into play the trapping at all the QDs visited by energy migration. The formation of charged QDs (i.e., trions) that amplify the AR, the effect of the electric field applied to bias the device, the efficient coupling with the hole and electron transport layers are other factors that must be taken into account. Systematic studies of how energy and charge transfer processes depend on composition and structure of surfaces and interfaces, taking also into account ensemble averages, are in high demand.

In this work, we present a detailed study into the optical absorption, fluorescence dynamics and quantum yield, as well as ultrafast transient absorption properties of $\mathrm{CdSe} / \mathrm{CdS}, \mathrm{CdSe} / \mathrm{Cd}_{0.5} \mathrm{Zn}_{0.5} \mathrm{~S}$, and $\mathrm{CdSe} / \mathrm{ZnS}$ QDs as a function of shell thickness while keeping constant the core radius and the organic ligand used to passivate the surface. Systematic studies like this give us the opportunity to check whether the fundamental knowledge of the QDs gained over a few decades of intense investigations allows us understanding the properties and behavior of QDs systems exhibiting a wide variety of structural and electronic features, going from quasi type-II $(\mathrm{CdSe} / \mathrm{CdS})$ to type-I with modulation of interface potential $\left(\mathrm{CdSe} / \mathrm{Cd}_{0.5} \mathrm{Zn}_{0.5} \mathrm{~S}\right.$, and $\left.\mathrm{CdSe} / \mathrm{ZnS}\right)$. Here, our attention is focused on the role of exciton trapping at surface and interface traps and on non-radiative Auger recombination in QDs carrying either net charges or multiple excitons, both phenomena being the likely cause of the decreased efficiency of electroluminescence compared with photoluminescence of QDs.

\section{TRAPPING AND PHOTOLUMINESCENCE DYNAMICS}

$\mathrm{CdSe} / \mathrm{Cd}_{\mathrm{x}} \mathrm{Zn}_{1-\mathrm{x}} \mathrm{S}$ QDs were synthesized following previously reported protocols. ${ }^{14} \mathrm{CdSe}$ cores were prepared following a modified procedure with respect to that described by van Embden et al. ${ }^{15}$. The shell growth and core-shell QDs synthesis was carried out in accordance with a modified SILAR protocol previously published. ${ }^{15}$ Oleylamine was chosen as capping agent, yielding good solubility in both chloroform and toluene. Specifically, $\mathrm{CdS}$ and $\mathrm{Cd}_{0.5} \mathrm{Zn}_{0.5} \mathrm{~S}$ (henceforth $\mathrm{CdZnS}$ ) shells were epitaxially grown on $3.8 \mathrm{~nm}$ CdSe core QDs, with shell thicknesses ranging from 1 to 6 monolayers (ML). Homogeneous $\mathrm{ZnS}$ shells, with thickness ranging from 1 to $5 \mathrm{ML}$, were grown on $4.2 \mathrm{~nm}$ CdSe core QDs. Therefore, in comparing the behavior of our QDs we are probing the effects of the composition and structure of the shells while keeping almost constant the core radius and the capping agent.

\subsection{General structure and electronic properties}

The series of CdSe/CdS QDs with increasing shell thickness from 1 to $6 \mathrm{ML}$ were verified to belong to the quasi type-II semiconductor nanocrystals, where holes are effectively confined in the core whereas electron wavefunctions spread over both the core and the shell. ${ }^{16}$ At the temperature reached during the CdS shell growth, the formation of a CdSe $\mathrm{S}_{1-\mathrm{x}}$ alloy in our $\mathrm{CdSe} / \mathrm{CdS}$ samples is revealed by surface enhanced Raman scattering experiments. ${ }^{17}$ This alloy has a twofold effect: (i) it reduces the formation of defects (e.g. dislocations) at the interface caused by the lattice mismatch between core and shell materials, thus it decreases the tendency of photoexcited carriers to localize around those defects; (ii) it smooths the confinement potential between core and shell, which contributes in the reduction of the non-radiative Auger mechanism for exciton relaxation. These features, together with the reduced Coulomb interactions between electrons and holes due to reduced wavefunction overlap, contribute to making $\mathrm{CdSe} / \mathrm{CdS}$ one of QDs exhibiting record high PLQY. Note, however, that the full achievement of this result requires growing thick CdS shells up to CdSe/CdS QDs known as giant-QDs. CdSe/CdZnS, and CdSe/ZnS QDs are classified as more conventional type-I structures inasmuch as the band edges of the shell encompass that of the core. This type of band alignment leads to the formation of potential barriers that effectively contain the extent of the electron and hole wavefunctions to within the core volume and away from the surface. ${ }^{18}$ Although this energy level configuration increases the surface passivation, with respect to $\mathrm{CdSe} / \mathrm{CdS}$ QDs, other effects come into play. The presence of sharp interfaces causes the formation of defects, due to lattice mismatch, hampering their optical properties. Moreover, this shape of the interface potential favors Auger recombinations.

\subsection{Phenomenological model for the PL decay}

Systematic measurements and analyses of the photoluminescence decay curves were carried out for the three series of

QDs, $\mathrm{CdSe} / \mathrm{CdS}, \mathrm{CdSe} / \mathrm{CdZnS}$, and $\mathrm{CdSe} / \mathrm{ZnS}$, with increasing number of ML composing the shell. These experiments 
aimed to clarify the effect of different core/shell interfaces on trapping dynamics. Indeed, trapping processes are intertwined with radiative recombination of excitons in QDs. As shown by Mooney and Scholes, ${ }^{19,20}$ traps are not static charge accumulators, because de-trapping process is possible, and energetically favored at room temperature. The detrapping process results in the regeneration of bound excitons, contributing to PL with a delayed dynamics, coherently with microsecond lasting PL observed. ${ }^{31}$ Hence radiative rate combine with trapping processes, resulting in multiexponential PL decays. Due to overlapping timescales, the conventional multi-exponential fitting analysis is not reliable in separating these contributions. We achieved an accurate untangling of these processes by formulating a kinetic model for the description of PL dynamics. The presented model reduces that proposed by Scholes ${ }^{20}$ to a phenomenological formulation, i.e. the rate constant introduced can be modified to suitably fit other systems. According to this model, different distributions of radially distributed traps interact with bound excitons, resulting in trapping and de-trapping. ${ }^{22}$ Therefore, a set of differential equations accounts for the time evolution of bound exciton population $\left(\rho_{X}\right)$ and trap states $\left(\rho_{i}\right)$, where the i index indicates the i-th trap distribution.

Equations read:

$$
\begin{aligned}
& \frac{d \rho_{X}(t)}{d t}=\sum_{i}\left(v_{d t, i} \rho_{i}(t)-\left(v_{t, i}+k_{N R}+k_{R}\right) \rho_{X}(t)\right) \\
& \frac{d \rho_{i}(t)}{d t}=v_{t, i} \rho_{X}(t)-v_{d t, i} \rho_{i}(t),
\end{aligned}
$$

where $v_{t, i}\left(v_{d t, i}\right)$ represent trapping (de-trapping) constant for the i-th trap distribution. Accordingly, radiative and nonradiative processes, described by $k_{R}$ and $k_{N R}$ respectively, and trapping deplete bound exciton population, whereas detrapping augment it.
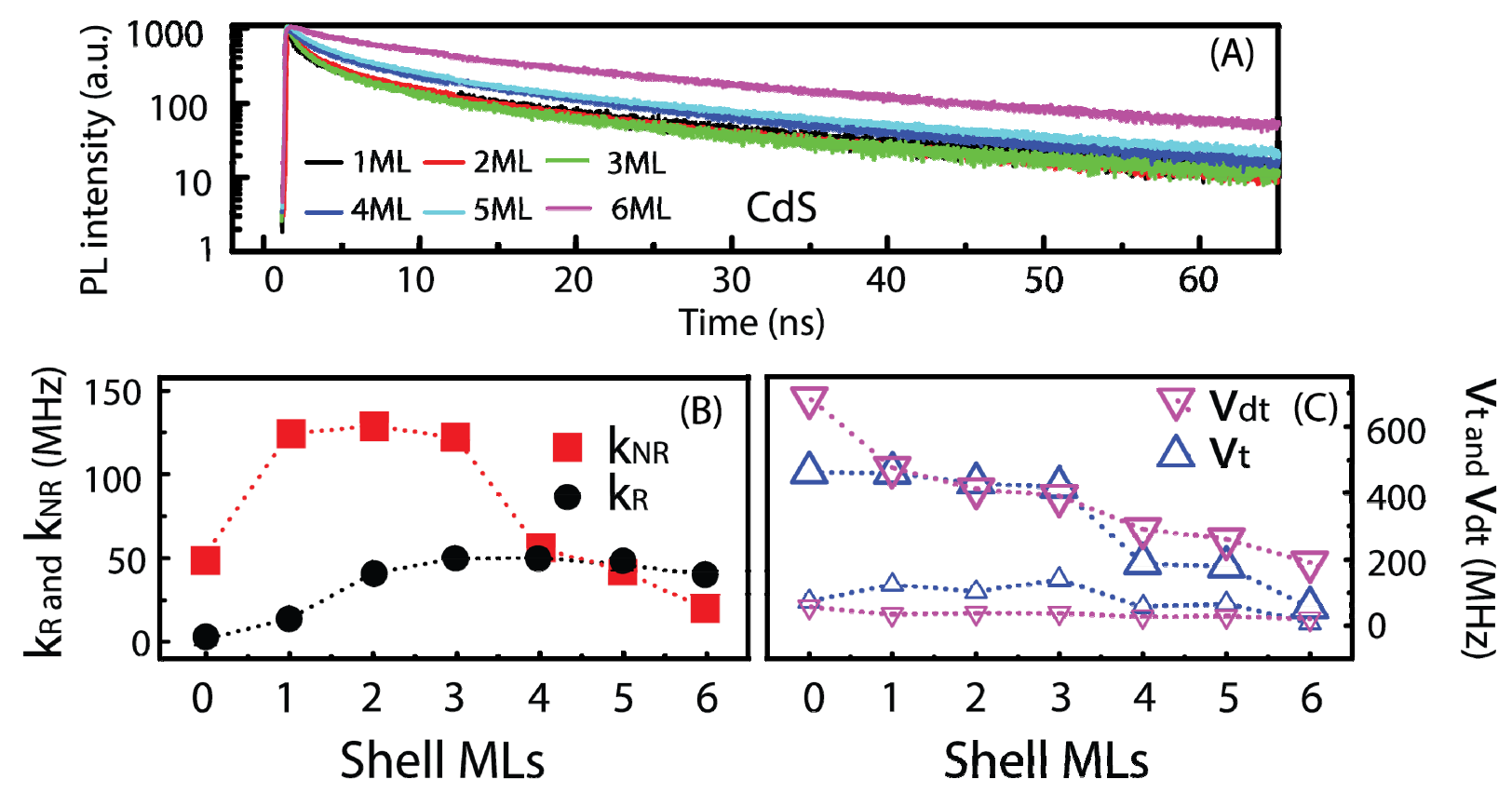

Figure 1- (A) Time resolved PL measurements for the CdSe/CdS series. (B) Non-Radiative (red squares) and radiative (black circles) rates obtained by model fitting of PL decay curve for CdSe/CdS series, lines are guide for the eye (C) Trapping (blue triangle) and detrapping (purple inverted triangle) rates obtained by model fitting of PL decay curve for CdSe/CdSe series, big triangle and small triangle indicate two different traps distributions. 
The PL decay curve $\mathrm{I}(\mathrm{t})$ is correlated with this population, through the radiative constant. Therefore, we performed a least square fitting procedure, minimizing the function:

$$
\chi=\int_{0}^{t}\left(I(t)-C * k_{R} \rho_{X}\left(\left[k_{R}, v_{t, i}, v_{d t, i}\right], t\right)\right)^{2} d t
$$

to extract rates for our samples. The rates included in this model were determined for the three different core/shell QDs, as shown in Figure 1 for $\mathrm{CdSe} / \mathrm{CdS}$ series. Notably, two trap distribution have been found necessary to fit the exciton population, and according to Jones calculations faster (slower) trap are ascribed to surface (interface) trapping. ${ }^{20}$ The presence of de-trapping accurately accounts for PL dynamics at long time delays. The behavior of trapping/detrapping rates reveals deep insights into the photophysics of shelling. ${ }^{22}$ Surface trapping rates display a decreasing trend with increasing shell thickness, due to reduced electronic coupling between bound exciton and trap states, as suggested by absorption spectra. This behavior is observed for each of the three series, confirming the good passivation of surfaces. In particular, CdZnS shell seems to provide the better passivation among the series, with $\mathrm{k}_{\mathrm{t}, 1}$ decreasing from 462 to $50 \mathrm{MHz}$, from bare core to 6ML thick shell. On contrary, interface trapping rates gain more importance in thick shell samples, due to the physical origin of interface traps, associated with lattice mismatch. Comparing different series, interface trapping is higher in $\mathrm{CdSe} / \mathrm{ZnS}$ with respect to $\mathrm{CdSe} / \mathrm{CdZnS}$ and $\mathrm{CdSe} / \mathrm{CdS}$, thereby confirming the detrimental formation of defects at the $\mathrm{CdSe} / \mathrm{ZnS}$ interface.

Finally, model fitting provided information on radiative constant, as well. Observed non-monotonous trend for the radiative constant in the $\mathrm{CdSe} / \mathrm{CdS}$ is explained by the concurrence of two counteracting effects. The formation of $\mathrm{CdSe}_{\mathrm{x}} \mathrm{S}_{1-\mathrm{x}}$ alloy induces an increase in effective volume, increasing $k_{R} \cdot{ }^{21}$ On the other hand, the quasi-Type II nature of $\mathrm{CdSe} / \mathrm{CdS}$ interface reduces electron-hole overlap, thereby reducing $k_{R}$. Therefore, the alloy formation up to the third ML induces an increase in the radiative constant. The saturation of the alloy, results in the expected attenuation of $k_{R}$.

\subsection{Trapping, De-trapping rates and Quantum Yield}

We measured PLQY for the three series of QDs, employing the relative method developed by Demas. ${ }^{32}$ The model formulated in Eq. 1 encompasses a non-radiative constant $\left(k_{N R}\right)$ to account for non-unitary PLQY values. Indeed, the net effect of trapping/de-trapping events is the complete regeneration of trapped excitons into emissive bound excitons. Therefore, lower PLQY imply the presence of other non-radiative processes. The presence of a time-dependent fraction of dark dots, observed in blinking and single molecule experiments, is believed to determine a non-unitary PLQY. ${ }^{33,34}$ The reported reversibility between dark and bright states suggests a tight link with above mentioned trapping-detrapping equilibrium. We suggest that accumulation of charges at trap sites, generating charged QDs in which Auger recombinations are strongly enhanced, could lay at heart of this phenomenon. ${ }^{23}$ With the aim of investigating these charged species, the subtle distinction between velocities and rate constants becomes crucial. The starting point is to describe trapping process as a chemical reaction between a bound exciton (X) and a neutral trap (n), generating a charged trap (c). Then, the trapping velocity is defined as a $v_{t, n}=k_{t, n} \rho_{X} \rho_{N}$, where $k_{t, n}$ is the trapping rate constant. Thence, a refined version of the phenomenological model reads:

$$
\begin{aligned}
& \frac{d \rho_{X}}{d t}=\sum_{i}\left(-k_{t, i} \rho_{X} \rho_{N, i}+k_{d t, i} \rho_{C}\right)-\left(k_{R}+k_{N R}\right) \rho_{X} \\
& \frac{d \rho_{N, i}}{d t}=-k_{t, i} \rho_{X} \rho_{N, i}+k_{d t, i} \rho_{C, i} \\
& \frac{d \rho_{c, i}}{d t}=k_{t, i} \rho_{X} \rho_{N, i}-k_{d t, i} \rho_{C, i} .
\end{aligned}
$$

Differently from PL decay measurements, PLQY measurements are based on steady state experiments, where QDs are continuously excited. Mathematically, this is described the application of steady state conditions the Eq. 3, from which the equivalence condition of trapping and detrapping velocities is obtained, namely: 


$$
v_{t, i}=k_{t, i} \rho_{X} \rho_{N}=v_{d t, i}=k_{d t, i} \rho_{C, i}
$$

Therefore, the accumulation of trapped charges can be described by the ratio between detrapping and trapping rates, which is referred as $R_{D T}$. Considering that, in steady state approximation $\rho_{X}$ is also constant, $R_{D T}$ is defined as:

$$
\frac{v_{d t}}{v_{t}} \propto \frac{k_{d t}}{k_{t}}=R_{D T}
$$

Noteworthy, this parameter obtained by model fitting of PL decay curves was found to correlate with PLQY value, as shown in Figure 2a. The proportionality constant may show some variation on increasing the number of shell monolayers due to changes in the ensemble averaged densities of empty and charged traps. Nevertheless, such correlation was observed for each different series. The direct relation between these two quantities is a proof of detrimental effect of charge accumulation. ${ }^{23}$

\section{EXCITATION ENERGY DEPENDENCE OF PL QUANTUM YIELD}

The systematic measurement of PLQY as a function of excitation photon energy was carried out for the three series of core/shell QDs. According to previous reports by Loomis and Kambhampati, ${ }^{24,25}$ PLQY has been found to strongly depend on the energy of excitation photon, as shown in Figure $2 b$. The absorption of highly energetic photons generates hot carriers, characterized by different carrier distribution. This prompt fast trapping rates toward higher-lying trap sites (HT), containing several vibrational quanta, as depicted in Figure 2c..$^{19,25,34}$
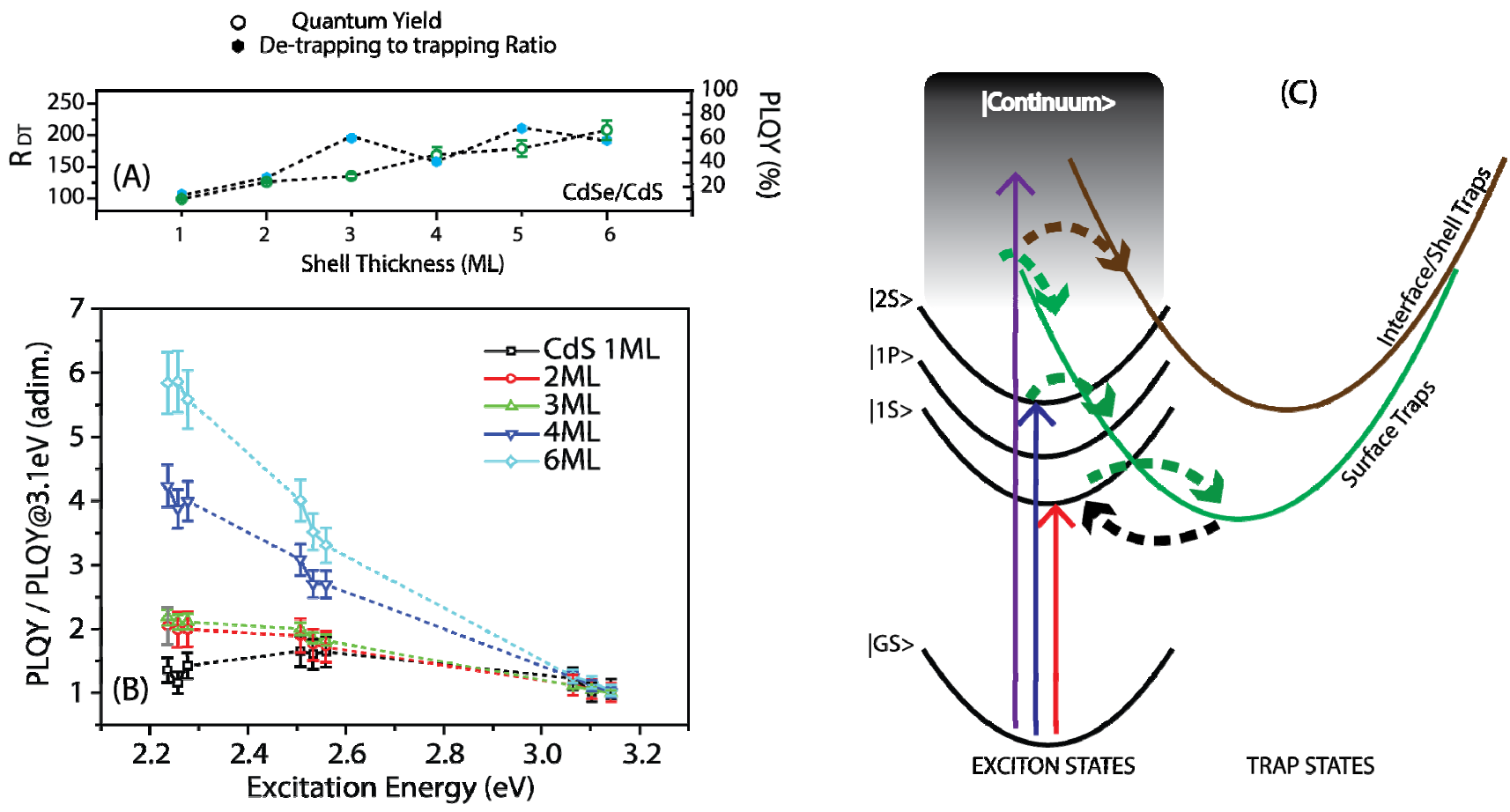

Figure 2- (A) Correlation between $\mathrm{R}_{\mathrm{DT}}$ parameter (cyan hexagon) obtained by model fitting of PL decay and measured PLQY (green circles) for CdSe/CdS. (B) Excitation energy dependence of PLQY, normalized on PLQY@3.1eV, for CdSe/CdS QDs series. (C)Depiction of hot carrier trapping in QDs. Hot carriers undergo fast trapping towards high-lying trap sites, which contain several quanta of vibration. The subsequent relaxation of trapped carrier unbalances the trapping-detrapping equilibrium. 
Trapping at HT unbalances trapping-detrapping equilibrium, due to its kinetic rather than thermodynamics nature. As demonstrated in the previous section, the balance between trapping and de-trapping rates determines the detrimental accumulation of trapped charges and the formation of charged QDs. Within this framework, excitation energy resolved PLQY measurements bridge the energetics and the dynamics of trapping process. Indeed, besides the energy distribution of trap states, the relation between trapping-detrapping equilibrium and PLQY need to be considered, i.e. charged QDs experience an enhancement of Auger recombinations. ${ }^{23}$

Figure $2 b$ shows how shell thickness modulates this excitation energy dependence (EED) of PLQY. Moreover, shell composition profoundly affects the observed EED. For CdSe/CdS and CdSe/CdZnS, a marked EED of PLQY is observed for thicker shell ( $>3 \mathrm{ML})$. The wavefunction of hot excitons created under the higher excitation energy $(3.1 \mathrm{eV})$ can reach and interact with surface traps in both $\mathrm{CdSe} / \mathrm{CdS}$ and $\mathrm{CdSe} / \mathrm{CdZnS}$ QDs. On the other hand, when excitation energy is lowered excitons are generated inside CdSe core and PLQY increases.

In contrast with expectations, the observed PLQY increase is higher for CdS than for $\mathrm{CdZnS}$ shell. For instance, at $6 \mathrm{ML}$ shell the increase of PLQY from 3.1 to $2.25 \mathrm{eV}$ is 6-fold for CdS, whereas it is only 3-fold for CdZnS. Being CdZnS bulk bandgap higher than $\mathrm{CdS}$ one ( 3 and $2.4 \mathrm{eV}$ respectively), the observed difference is ascribed to a different nature of the hetero-interface: the smoothness of $\mathrm{CdSe} / \mathrm{CdS}$ ensure a lower interface defectivity, whereas the abruptness of $\mathrm{CdSe} / \mathrm{CdZnS}$ interface is likely to nucleate interface defects, acting as HT sites. Therefore, despite the higher confining potential, surface defect passivation is counteracted by interface defect creation in CdZnS, as well.

The harmfulness of interface defects is clearer when analyzing EED of PLQY for ZnS series. The high lattice mismatch between CdSe and $\mathrm{ZnS}$ induces a dislocated growth ${ }^{17}$ and a non-monotonous trend is observed with increasing shell thickness. Indeed, the steepest increase of PLQY from 3.1 to $2.25 \mathrm{eV}$ has been observed for 2ML. At shell thickness above 3ML, the nucleation of interface defects augment the number of HT and the EED of PLQY, together with its absolute value, are lowered.

\section{FAST NONRADIATIVE DECAY OF EXCITONS}

The building-up of charge populations at surfaces in photoexcited QDs, also referred as photocharging, was reported first by Klimov for PbSe QDs. ${ }^{26}$ Charged exciton states were found to present efficient Auger relaxation channel, leading to a quenching of PL. Notably, this effect was observed preferentially under highly energetic excitation, suggesting a link with the above reported EED of PLQY.

The kinetic model presented in the previous section encompasses the presence of these processes and includes it into the $\mathrm{k}_{\mathrm{nr}}$ parameter. To directly observe these fast relaxations and investigate the role played by different shells, the three series of QDs in toluene solution were studied by multicolor TA technique, using $400 \mathrm{~nm}$ excitation and white light probe.

TA dataset are two dimensional maps, resolved in both wavelength and time, as shown in Figure 3. The differential absorption mainly originates from state filling, and all signal present were successfully explained on the basis of QDs excitonic structure. ${ }^{27}$ As reported in Figure 3a, three main bleaching signal observed are assigned to corresponding core exciton transitions. Moreover, the high pump fluence is expected to generate different species, such as multi-excitons. 

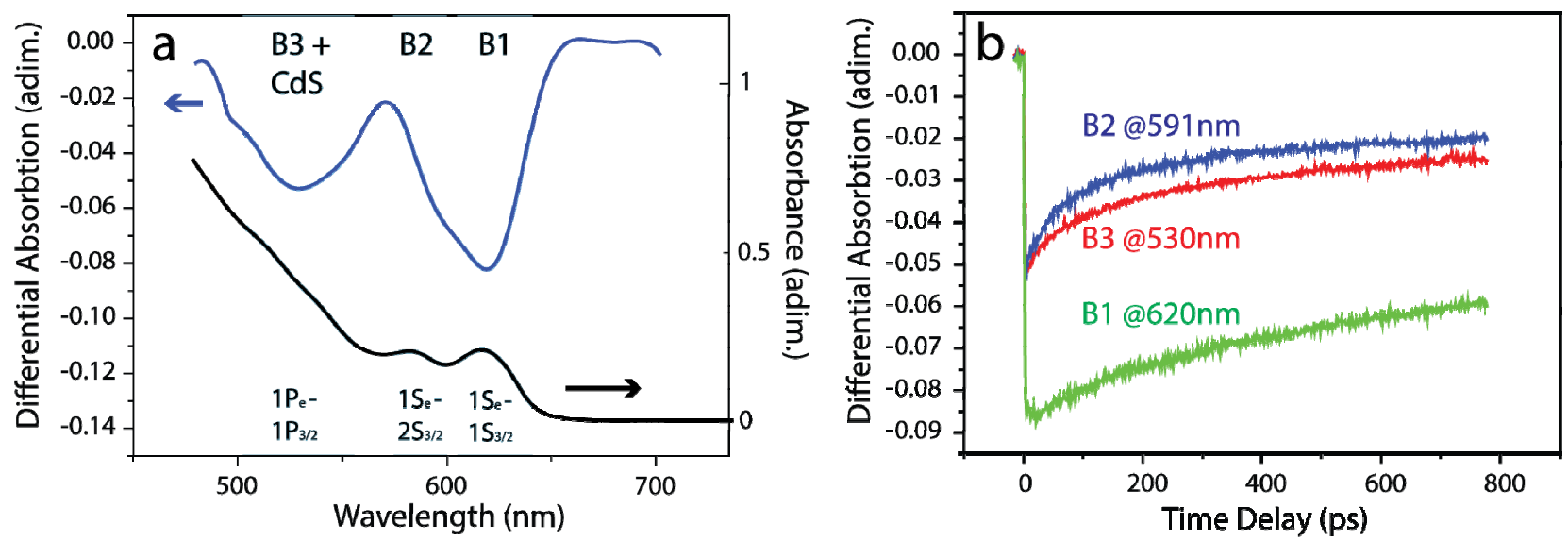

Figure 3- (A) Fixed time delay (2ps) section of the TA spectrum (blue) recorded for CdSe/CdS 2ML under intense excitation, generating 1.8 average excitons per dot. The spectral structure is correlated with linear absorption spectrum (black). (B) Bleaching recovery of main signals B1 (green), B2 (blue) and B3 (red), obtained taking fixed wavelength section of the twodimesional dataset.

Global analysis procedures allow unveiling the nature of these signals, where different species and overlapping features concur in determining a complex spectrum. Therefore, we employed singular value decomposition (SVD) analysis to disentangle different species and their recombination dynamics. SVD method decomposes the $2 \mathrm{D}$ dataset $\Delta A(\lambda, t)$ in a collection of principal spectra $f_{S}(\lambda)$ and decay curves $I_{S}(t),{ }^{29}$ which reconstruct TA data as $\Delta A(\lambda, t)=\sum_{S} f_{S}(\lambda) \times$ $I_{S}(t)$.

This analysis is useful to identify the independently evolving species contributing to the spectrum (Figure 4). At least three species were found to contribute significantly to TA spectra of each QDs studied. Similar results were reported by Kagan. ${ }^{30}$ According to their signals and recombination dynamics, shown in Figure $4 \mathrm{a}$ and $4 \mathrm{~b}$ respectively, we assigned them to single exciton, bi-exciton and un-relaxed excitons, respectively.
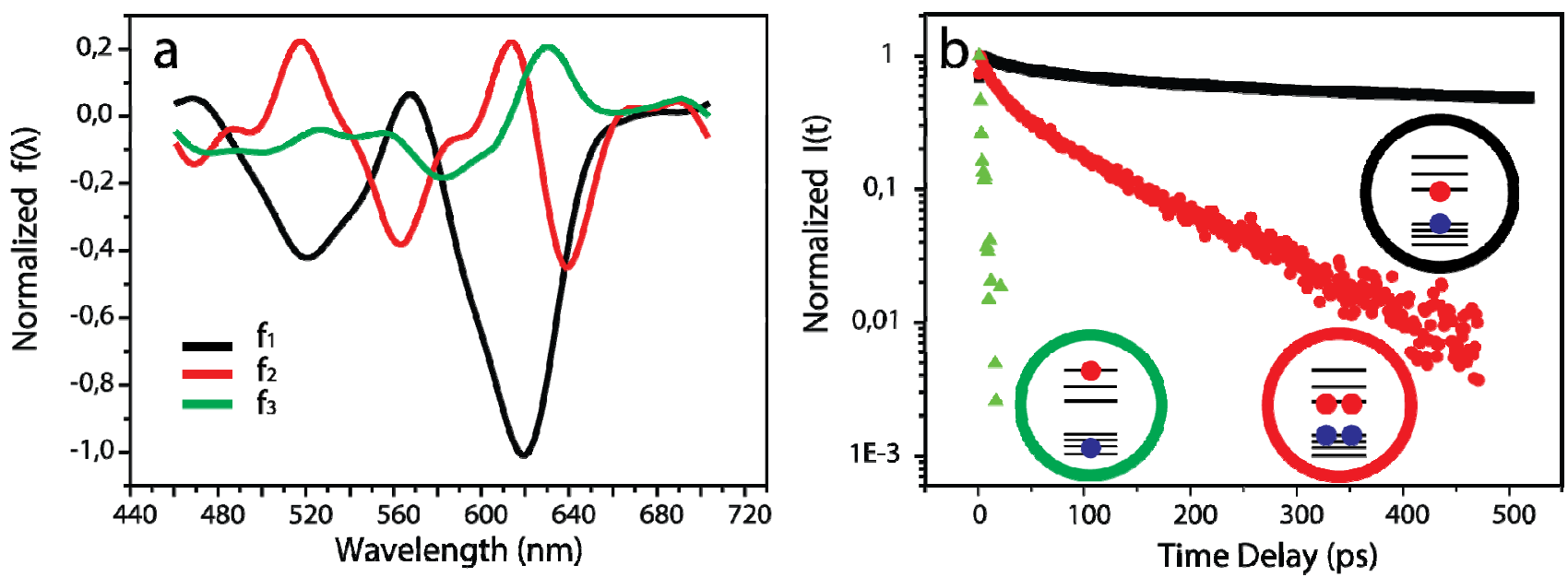

Figure 4- (A) Normalized principal spectra $f(\lambda)$, obtained by SVD analysis on CdSe/CdS $2 M L$ TA spectrum (1.8 average exciton occupancy). (B) Corresponding decay profiles $I(t)$ and their species assignment. 
Indeed, the first principal spectrum (black curve in Figure 4a) display the slowest decay, throughout the temporal window under investigation (1-800ps). Its persistence at long time delays $\mathrm{t}>400 \mathrm{ps}$, when all multi-excitonic effects are expected to be concluded, suggests a safe assignment to singly excited dots. ${ }^{27,28}$ On the other hand, the red-shifted spectral structure and faster decays indicate that the second principal spectrum (red curve in Figure 4a) is related to biexciton species. The third principal spectrum (green curve in Figure 4a) presents a red-shifted curve and lasts few picoseconds. Therefore, we assign it to hot exciton relaxation processes.

Table 1-Average lifetime for first principal spectra, obtained by multi-exponential fitting of the first decay profile

\begin{tabular}{cccccc} 
& \multicolumn{3}{c}{$<\mathrm{l}_{1}>(\mathrm{ps})$} \\
\cline { 2 - 3 } & $\mathrm{CdS}$ & & $\mathrm{CdZnS}$ & & $\mathrm{ZnS}$ \\
\cline { 2 - 3 } \cline { 5 - 6 } Cores & 134 & & 134 & & \\
$1 \mathrm{ML}$ & 296 & & 369 & & 342 \\
$2 \mathrm{ML}$ & 314 & & 278 & & 294 \\
$3 \mathrm{ML}$ & 564 & & 390 & & 245 \\
$4 \mathrm{ML}$ & 356 & & 423 & & 264 \\
$5 \mathrm{ML}$ & & & & & 200 \\
$6 \mathrm{ML}$ & 531 & & 621 & & \\
\hline
\end{tabular}

We carried out a preliminary analysis of the decay profiles associated with the first principal spectra, with the aim of investigating photo-charging effects on core/shell QDs. Their average lifetime, calculated by multi-exponential fitting, is reported in Table I. For $\mathrm{CdSe} / \mathrm{CdS}$ and $\mathrm{CdSe} / \mathrm{CdZnS}$, single exciton recombinations appear to be slowed down with increasing shell thickness. This effect is mostly due to the passivation of the surfaces and to the shifting of surface traps away from CdSe core. Indeed, although charge accumulation takes place, charged trap sites are shifted away from core excitons and Auger recombinations are slowed down. Single excitons in $\mathrm{CdSe} / \mathrm{CdS}$ QDs display longer lifetimes, with respect to those in $\mathrm{CdSe} / \mathrm{CdZnS}$ QDs. This further proofs the beneficial effect of the smoothing of interfaces, caused by $\mathrm{CdSe}_{1-\mathrm{x}} \mathrm{S}_{\mathrm{x}}$ alloying.

The effect of interfacial traps on fast recombination is once more evident in $\mathrm{CdSe} / \mathrm{ZnS}$ series. The observed single exciton lifetime decreases with increasing shell thickness along this series. Once more, this is ascribed to the nucleation of interfacial defects. From a quantitative point of view, the observed recombination cannot be compared with those estimated by kinetic modeling of PL decays. Indeed, these experiments are performed under very different excitation intensity conditions. As shown by Klimov, excitation fluence strongly affect the photocharging effect and therefore results in different dynamics.

\section{CONCLUDING REMARKS}

The effect of charge trapping and accumulation at surfaces was investigated for different core/shell QDs: CdSe/CdS, $\mathrm{CdSe} / \mathrm{CdZnS}$, and $\mathrm{CdSe} / \mathrm{ZnS}$. An in-depth analysis was carried out through time-resolved PL, PLQY and transient absorption measurement. The photophysics of QDs was successfully and exhaustively described by a kinetic model, comprising both trapping and de-trapping processes. Both shell composition and thickness were found to play a pivotal role in modulating these processes, which dictate their efficiency in device applications. The interplay between three factors was found to dictate QDs performances: the smoothness of interface potential, the surface passivation, and the nucleation of interface defects.

Whenever dealing with core/shell systems, we suggest to consider these three factors, in order to achieve desired properties. A delicate balance is necessary: for instance, although a $\mathrm{ZnS}$ shell provides an optimal surface passivation, the sharp interface potential and the massive nucleation of interface defects hamper their performances.

On the other hand, CdS provides a smooth and defect free interface, unfortunately resulting in a poor surface passivation, which turns out to be sufficient only in case of thick shells. 


\section{REFERENCES}

[1] Zhou, J.; Yang, Y.; Zhang, C.-y., Toward Biocompatible Semiconductor Quantum Dots: From Biosynthesis and Bioconjugation to Biomedical Application. Chemical Reviews 2015, 115, 11669-11717.

[2] Carey, G. H.; Abdelhady, A. L.; Ning, Z.; Thon, S. M.; Bakr, O. M.; Sargent, E. H., Colloidal Quantum Dot Solar Cells. Chemical Reviews 2015, 115, 12732-12763.

[3] Klostranec, J. M.; Chan, W. C. W., Quantum Dots in Biological and Biomedical Research: Recent Progress and Present Challenges. Advanced Materials 2006, 18, 1953-1964.

[4] Freeman, R.; Finder, T.; Willner, I., Multiplexed Analysis of $\mathrm{Hg}^{2+}$ and $\mathrm{Ag}^{+}$Ions by Nucleic Acid Functionalized CdSe/ZnS Quantum Dots and Their Use for Logic Gate Operations. Angewandte Chemie International Edition 2009, 48, 7818-7821.

[5] Yuan, M.; Liu, M.; Sargent, E. H., Colloidal Quantum Dot Solids for Solution-Processed Solar Cells. Nature Energy 2016, $1,16016$.

[6] Claussen, J. C.; Hildebrandt, N.; Susumu, K.; Ancona, M. G.; Medintz, I. L., Complex Logic Functions Implemented with Quantum Dot Bionanophotonic Circuits. ACS Applied Materials \& Interfaces 2014, 6, 3771-3778.

[7] Osborne, M. A.; Fisher, A.A.E., Charge-tunnelling and self-trapping: common origins for blinking, grey-state emission and photoluminescence enhancement in semiconductor quantum dots. Nanoscale 2016, 8, 9272.

[8] Gong, K.; Kelley, D.F., Surface Charging and Trion Dynamics in CdSe-Based Core/Shell Quantum Dots. J. Phys. Chem. C, 2015, 119, 637-9645.

[9] Walsh, B.R.; Saari, J.I.; Krause, M.M.; Nick, R.; Coe-Sullivan, S.; Kambhampati, P., Surface and interface effects on non-radiative exciton recombination and relaxation dynamics in $\mathrm{CdSe} / \mathrm{Cd}, \mathrm{Zn}, \mathrm{S}$ nanocrystals. Chemical Physics 2016, 471, 11-17.

[10] Shirasaki, Y.; Supran, G.J.; Bawendi, M.G.; Bulovic, V, Emergence of colloidal quantum-dot light-emitting technologies. Nature Photonics 2013, 7, 13-23.

[11] Pal, B.N.; Ghosh, Y.; Brovelli, S.; Laocharoensul, K.; Klimov, V.; Hollingsworth. J.A.; Htoon, H., 'Giant' $\mathrm{CdSe} / \mathrm{CdS}$ Core/Shell Nanocrystal Quantum Dots As Efficient Electroluminescent Materials: Strong Influence of Shell Thickness on Light-Emitting Diode Performance. NanoLett. 2012, 12, 331-336.

[12] Mashford, B.S.; Stevenson, M; Popovic, Z.; Hamilton, C.; Zhou, Z.; Breen, C.; Steckel, J; Bulovic, V; Bawendi, M.; Coe-Sullivan, S.; Kazlas, P.T., High-efficiency quantum-dot light-emitting devices with enhanced charge injection. Nature Photonics 2013, 7, 407-412.

[13] Yang,Y.; Zheng, Y.; Cao, W.; Titov, A.; Hyvonen, J.; Manders, J.R.; Xue, J.; Holloway, P.H.; Qian, L., Highefficiency light-emitting devices based on quantum dots with tailored nanostructures. Nature Photonics 2015, 9, 259266.

[14] Fortunati, I.; Signorini, R.; Bozio, R.; Jasieniak, J. J.; Antonello, A.; Martucci, A.; Giustina, G. D.; Brusatin, G.Guglielmi, M., Cdse Core-Shell Nanoparticles as Active Materials for up-Converted Emission. The Journal of Physical Chemistry C 2011, 115, 3840-3846.

[15] van Embden, J.; Jasieniak, J.Mulvaney, P., Mapping the Optical Properties of Cdse/Cds Heterostructure Nanocrystals: The Effects of Core Size and Shell Thickness. Journal of the American Chemical Society 2009, 131, 14299-14309.

[16] Boldt, K.; Kirkwood, N.; Beane, G.A.; Mulvaney, P. Synthesis of highly luminescent and photo-stable, graded shell $\mathrm{CdSe} / \mathrm{CdxZn1-xS}$ nanoparticles by in situ alloying. Chem Mater 20

$13,25,4731-4738$.

[17] Todescato, F.; Minotto, A.; Signorini, R.; Jasieniak, J.J.; Bozio, R. Investigation into the heterostructure interface of CdSe-based core-shell quantum dots using surface-enhanced raman spectroscopy. Acs Nano 2013, 7, 6649-6657.

[18] Chaudhuri, R.G.; Paria, S. Core/shell nanoparticles: Classes, properties, synthesis mechanisms, characterization, and applications. Chem Rev 2012, 112, 2373-2433.

[19] Mooney, J.; Krause, M. M.; Saari, J. I.; Kambhampati, P., Challenge to the Deep-Trap Model of the Surface in Semiconductor Nanocrystals. Physical Review B 2013, 87, 081201. 
[20] Jones, M.; Lo, S. S.; Scholes, G. D., Quantitative Modeling of the Role of Surface Traps in CdSe/CdS/ZnS Nanocrystal Photoluminescence Decay Dynamics. Proceedings of the National Academy of Sciences 2009, 106, 3011-3016.

[21] Gong, K.; Zeng, Y.; Kelley, D. F., Extinction Coefficients, Oscillator Strengths, and Radiative Lifetimes of CdSe, CdTe, and CdTe/CdSe Nanocrystals. The Journal of Physical Chemistry C 2013, 117, 20268-20279.

[22] Minotto, A.; Todescato, F.; Fortunati, I.; Signorini, R.; Jasieniak, J. J.; Bozio, R., Role of Core-Shell Interfaces on Exciton Recombination in CdSe-CdxZn1-xS Quantum Dots. The Journal of Physical Chemistry C 2014, 118, 24117-24126.

[23] Righetto, M.; Minotto, A.; Bozio, R., Bridging Energetics and Dynamics of Exciton Trapping in Core-Shell Quantum Dots. The Journal of Physical Chemistry C 2017, 121 (1), 896-902

[24] Hoy, J.; Morrison, P. J.; Steinberg, L. K.; Buhro, W. E.; Loomis, R. A., Excitation Energy Dependence of the Photoluminescence Quantum Yields of Core and Core/Shell Quantum Dots. The Journal of Physical Chemistry Letters 2013, 4, 2053-2060.

[25] Kambhampati, P., Hot Exciton Relaxation Dynamics in Semiconductor Quantum Dots: Radiationless Transitions on the Nanoscale. The Journal of Physical Chemistry C 2011, 115, 22089-22109.

[26] McGuire, J. A.; Sykora, M.; Robel, I.; Padilha, L. A.; Joo, J.; Pietryga, J. M.; Klimov, V. I., Spectroscopic Signatures of Photocharging due to Hot-Carrier Transfer in Solutions of Semiconductor Nanocrystals under LowIntensity Ultraviolet Excitation. ACS Nano 2010, 4, 6087-6097.

[27] Klimov, V. I., Spectral and Dynamical Properties of Multiexcitons in Semiconductor Nanocrystals. Annual Review of Physical Chemistry 2007, 58, 635-673.

[28] Kambhampati, P., Multiexcitons in Semiconductor Nanocrystals: A Platform for Optoelectronics at High Carrier Concentration. The Journal of Physical Chemistry Letters 2012, 3, 1182-1190.

[29] Ruckebusch, C.; Sliwa, M.; Pernot, P.; de Juan, A.; Tauler, R., Comprehensive data analysis of femtosecond transient absorption spectra: A review. Journal of Photochemistry and Photobiology C: Photochemistry Reviews $2012,13,1-27$.

[30] Turk, M. E.; Vora, P. M.; Fafarman, A. T.; Diroll, B. T.; Murray, C. B.; Kagan, C. R.; Kikkawa, J. M., Ultrafast Electron Trapping in Ligand-Exchanged Quantum Dot Assemblies. ACS Nano 2015, 9, 1440-1447.

[31] Rabouw, F. T.; Kamp, M.; van Dijk-Moes, R. J. A.; Gamelin, D. R.; Koenderink, A. F.; Meijerink, A.; Vanmaekelbergh, D., Delayed Exciton Emission and Its Relation to Blinking in CdSe Quantum Dots. Nano Letters $2015,15,7718-7725$.

[32] Crosby, G. A.; Demas, J. N., Measurement of Photoluminescence Quantum Yields. Review. The Journal of Physical Chemistry 1971, 75, 991-1024.

[33] Orfield, N. J.; McBride, J. R.; Wang, F.; Buck, M. R.; Keene, J. D.; Reid, K. R.; Htoon, H.; Hollingsworth, J. A.; Rosenthal, S. J., Quantum Yield Heterogeneity among Single Nonblinking Quantum Dots Revealed by Atomic Structure-Quantum Optics Correlation. ACS Nano 2016, 10, 1960-1968.

[34] Mooney, J.; Krause, M. M.; Kambhampati, P., Connecting the Dots: The Kinetics and Thermodynamics of Hot, Cold, and Surface-Trapped Excitons in Semiconductor Nanocrystals. The Journal of Physical Chemistry C 2014, $118,7730-7739$. 